\title{
Clics y comentarios como expresiones del dilema de intereses de los usuarios ante las noticias: el caso del agregador de noticias de lengua hispana Menéame
}

\section{Clicks and comments as expressions of the dilemma of users' news interests: the case of the Spanish-language social news aggregator, Menéame}

Begoña Zalbidea Bengoa. Universidad del País Vasco/Euskal Herriko Unibertsitatea. España. bego.zalbidea@ehu.eus

$[\underline{\mathrm{CV}}]$ (1) $\mathrm{G}$

Santiago Urrutia. Universidad del País Vasco/Euskal Herriko Unibertsitatea. España. santi.urrutia@ehu.eus

$[\mathrm{CV}](1) \mathrm{G}$

Idoia Camacho-Markina. Universidad del País Vasco/Euskal Herriko Unibertsitatea. España. idoia.camacho@ehu.eus

[CV] (B)

José María Pastor. Universidad del País Vasco/Euskal Herriko Unibertsitatea. España.

josemari.pastor@ehu.eus

$[\underline{\mathrm{CV}}] \odot \mathrm{G}$

Este artículo es producto de los proyectos de Investigación titulados "Reacciones desde la ética ciudadana en la Red Social Menéame, ante los contenidos de los medios de comunicación convencionales y sociales en España", y "Ética, información y redes sociales", referencias CSO2014-59077-R y GIU15/27, financiados por el Ministerio de Economía y Competitividad y la Universidad del País Vasco/Euskal Herriko Unibertsitatea, respectivamente.

Cómo citar este artículo / Referencia normalizada

Zalbidea, B., Urrutia, S., Camacho-Markina. I. y Pastor, J. M. (2020). Clics y comentarios como expresiones del dilema de intereses de los usuarios ante las noticias: el caso del agregador de noticias de lengua hispana Menéame. Revista Latina de Comunicación Social, 75, 327-339. https://www.doi.org/10.4185/RLCS-2020-1429

\section{RESUMEN}

Introducción. El estudio tiene como objetivo analizar la diversidad de intereses de los usuarios de las redes sociales identificando qué tipo de información visitan las audiencias y qué tipo de noticias comentan. Con este fin, se investigó la actividad de los usuarios del agregador de noticias en español de más éxito, la red social Menéame, examinando el comportamiento de la audiencia con respecto al contenido publicado en la red. Metodología. La investigación se llevó a cabo utilizando una muestra de 3.720 noticias seleccionadas al azar de un total de 51.520 artículos publicados en la portada de Menéame durante más de cinco años (2010-2015). Se analizó el número de visitas y comentarios que 
recibió cada ítem, y las dos variables, clics y comentarios, se relacionaron con el tipo de contenido y sus categorías temáticas. Resultados. Los resultados muestran que existe una clara oposición entre clics y comentarios en términos de tipos de contenido, con dos tendencias principales con respecto a las noticias duras y blandas. Por un lado, en promedio, estos últimos reciben casi 4.000 visitas más que los artículos de temática dura, que en promedio reciben alrededor de 5.500 clics. Sin embargo, las noticias duras tienden a provocar una mayor cantidad de comentarios que las noticias blandas. El promedio para el primero es de 66 comentarios, mientras que las noticias suaves reciben 10 menos $(55,7)$. Discusión y conclusiones. Los datos sugieren que los clics y los comentarios expresan dos intereses principales de los usuarios frente a las noticias y que son opuestos y complementarios.

PALABRAS CLAVE: usuarios; clics; comentarios; noticias duras; noticias blandas.

\begin{abstract}
Introduction. The study aims to analyse the diversity of the interests of users of social networks by identifying what kind of information audiences visit and what kind of news they comment on. To this end, the activity of the users of the most successful Spanish-language news aggregator, the Menéame social network, was investigated, by examining audience behaviour with respect to the content published on the network. Methodology. The research was carried out using a sample of 3,720 news items randomly selected from a total of 51,520 items published on the front page of Menéame throughout more than five years (2010-2015). The number of visits and comments that each item received was analysed, and the two variables, clicks and comments, were related to the type of content and its thematic categories. Results. The results show that there is a clear opposition between clicks and comments in terms of content types, with two main trends with respect to hard and soft news items. On the one hand, on average the latter receive almost 4,000 visits more than hard items, which on average receive around 5,500 clicks. However, hard news tends to provoke a greater amount of comments than soft news. The average for the former is 66 comments, while soft news receives 10 less (55.7). Discussion and conclusions. Data suggest that clicks and comments express two main interests of users in front of news and that they are both opposing and complementary.
\end{abstract}

KEYWORDS: users; clicks; comments; hard news; soft news.

\title{
CONTENIDO
}

1. Introducción. 2. Metodología. 3. Resultados. 3.1. Qué tipo de noticias visitan más los usuarios. 3.2. Qué tipo de noticias comentan más los usuarios. 3.3. Relación entre clics y comentarios. 4. Discusión y conclusiones. 5. Referencias bibliográficas.

Artículo redactado originalmente en inglés por el equipo de investigación y revisado por el traductor Robert Curwen.

\section{Introducción}

Una gran cantidad de investigaciones realizadas a lo largo de la última década ha hallado que existe disparidad de intereses entre los periodistas y el público con respecto a las noticias. Se observa reiteradamente que los primeros muestran preferencia por las llamadas "noticias duras", mientras que los lectores buscan, en cambio, noticias blandas. Los investigadores llegan a esa conclusión tomando en cuenta como indicador del interés de la audiencia el número de visitas o clics recibidos por las noticias. En este estudio queremos analizar si hay que persistir en mantener esta idea, o si es necesario cambiarla y concluir que el comportamiento del público y los usuarios frente a las noticias es más complejo de lo que se pensaba. 
En ese sentido, en los últimos años se ha empezado a utilizar también otro factor para medir el interés de los lectores aparte de los clics, a saber, el número de comentarios. En base a este indicador, el público otorga más importancia a las cuestiones públicas en comparación con lo que indicaban las mediciones anteriores. En esta investigación queremos analizar la diversidad de intereses de los usuarios mediante el examen de ambos indicadores, clics y comentarios, e intentar vislumbrar el trasfondo de cada uno de ellos.

Partimos de la idea de que ambos elementos son expresiones de dos tipos de intereses de los usuarios frente a las noticias. Por ello, analizaremos qué tipo de contenidos visitan más y comentan más los usuarios para comprobar si existen determinados tipos de informaciones que tienen probabilidad de ser más consumidas, a la vez que otras categorías de noticias podrían revelar una tendencia a ser más comentadas.

Para llevar a cabo esta tarea, la investigación analiza los clics y los comentarios utilizando datos provenientes del agregador de noticias más importante de habla hispana, la red social Menéame. Este sitio web comenzó a funcionar a finales de 2005, después del lanzamiento de Digg, pero en la actualidad se parece más a Reddit, y tiene alrededor de 9 millones de visitantes únicos al mes (Martínez, 2017). Los usuarios pueden publicar un texto con un enlace al origen de una noticia e intentar promocionarla a la página principal buscando los votos del resto de usuarios. Si la propuesta obtiene un cierto número de votos, las noticias aparecerán en la portada del sitio. Estas piezas informativas son consideradas en la red como las más relevantes en cada momento, por lo que se convierten en las únicas noticias leídas por la mayoría de usuarios.

Para desarrollar el estudio partimos de una muestra probabilística de 3.720 noticias correspondientes a más de cinco años desde que comenzó a funcionar el contador de clics en la red Menéame (desde septiembre de 2010 hasta diciembre de 2015). La investigación analiza qué tipo de noticias visitan más los usuarios y cuáles son las más comentadas.

Los resultados muestran que los usuarios prefieren visitar noticias blandas, a la vez que comentan más las noticias duras. Para comprender y explicar este resultado, el marco teórico del estudio involucra varios aspectos y examina la naturaleza de los intereses frente a las noticias, presentando su diversidad. En el apartado de Discusión tratamos de avanzar algunas ideas sobre las implicaciones de esta realidad para el ámbito del periodismo y el de los medios de comunicación.

El interés del público frente a las noticias puede analizarse desde dos perspectivas, aunque ambas están estrechamente entrelazadas: puede ser considerado como algo que se intenta promocionar desde el exterior entre los lectores, o como algo que tiene su origen en la actitud del propio público. Para analizar la primera perspectiva podemos recurrir a la tradición de la "agenda setting" mientras que, para la segunda, proponemos tener en cuenta la teoría de usos y gratificaciones.

La teoría de la "agenda setting" ha sido utilizada en una gran cantidad de estudios y señala que los medios tienen el poder de establecer la agenda pública (Carazo, 2018, p. 17). Subraya que el público toma en cuenta y concede relevancia a los problemas o temas que previamente difunden las organizaciones de noticias. Desde este punto de vista, los medios han tenido tradicionalmente la capacidad de divulgar los temas que la audiencia tiene en cuenta y debate en un momento determinado. En este sentido, se dice que los medios online promueven agendas que son en gran medida comparables a los medios de comunicación tradicionales (McCombs, 2005, p. 545).

Si ubicamos la fuente del interés en la propia actitud del público, la expansión de la comunicación a través de la red ha relanzado la importancia de la teoría de los usos y gratificaciones (Ruggiero, 
2000, p. 3). Un principio importante de esta teoría del comportamiento de la audiencia es que el uso de los medios es selectivo y está motivado por la autoconciencia racional de las propias necesidades del individuo y la expectativa de que esas necesidades serán satisfechas por unos tipos particulares de medios y contenidos (Katz et al., 1974, p. 30). Con respecto a nuestro estudio, las noticias relacionadas con temas socio-políticos pueden conciliar un tipo de actividad de los usuarios que les lleve a participar más en el debate mediante la realización de comentarios, mientras que las noticias relacionadas con el entretenimiento podrían tener una tendencia a atraer solo clics.

Las necesidades mencionadas por la teoría de los usos y las gratificaciones pueden estar estrechamente relacionadas con los "intereses", los cuales pueden clasificarse como "intereses para usar los medios de comunicación con objetivos reseñables" e "intereses para usarlos como evasión". Con respecto a la primera categoría, los investigadores hallaron, por ejemplo, que un tipo concreto de medios, los diarios tradicionales, satisfacían la necesidad de integración política (Katz, Gurevitch y Haas, 1973, p. 2). En nuestro caso tenemos que ver si los comentarios de los usuarios, en cuanto que suponen una mayor actividad por parte de los mismos, pueden estar especialmente relacionados con asuntos públicos. Por otro lado, la gente usa los medios de comunicación para divertirse y entretenerse (Katz y Foulkes, 1962, p. 377). Podemos abordar el análisis de clics desde este punto de vista, para examinar si están más vinculados con noticias de evasión.

Aunque las nuevas tecnologías se consideran un elemento clave para incrementar la participación, inmediatamente se subrayan las diferencias que se producen en el nivel de actividad de los usuarios. Muchas investigaciones, basadas en la información obtenida a partir de los contadores de clics y el número de contenidos compartidos y comentarios realizados por los usuarios, han encontrado que la cantidad de quienes comentan los artículos de noticias es relativamente pequeña en comparación con el número de los que las leen (Diakopoulos y Naaman, 2011, 3). Estos autores hallaron en su estudio sobre el sitio web de un periódico californiano que la mayoría de los comentarios fueron realizados por un grupo muy pequeño de participantes, por lo que la distribución de frecuencia de comentarios entre usuarios estaba muy escorada. Otros investigadores llegaron a la misma conclusión en otro estudio, y reiteraron que solo una pequeña parte de los usuarios de una red comparte información o comentarios sobre el contenido, mientras que la gran mayoría simplemente visita el sitio (Karlsson et al., 2015). A partir de este hallazgo, es equivocado pensar que el acceso a las tecnologías digitales convierte automáticamente a las personas en participantes activos (Van Dijck, 2009).

Este punto de vista se ha resumido en una disposición básica llamada "regla del 1\%" (Arthur, 2006). Solo el 1\% de las personas crea contenido, el 10\% participa mediante comentarios o contribuciones y el otro 89\% únicamente lo consume (Arthur, 2006). Además, los usuarios más activos suelen tener varias particularidades relacionadas con su ideología y género, lo cual les diferencia del resto de usuarios (Friemel y Dötsch, 2015).

Sin embargo, utilizando datos de encuestas sobre una población más amplia de usuarios de medios online de seis países diferentes, varios investigadores han indicado que la actividad de los mismos es mucho mayor que la señalada anteriormente. Dicen que comentar y compartir noticias en la mayoría de los países está significativamente más extendido que la llamada "regla del 1\%". Una minoría significativa de usuarios de Internet toma parte activa en las noticias e información y, en particular, comentar y compartir a través de las redes sociales es algo muy extendido (Kalogeropoulos et al., 2017, pp. 1-2).

En su estudio, estos autores descubrieron lo siguiente: (1) las personas que usan una gran cantidad de plataformas de redes sociales diferentes y usan las mismas para compartir noticias son significativamente más propensas a involucrarse activamente fuera de las redes sociales realizando 
comentarios en sitios web de noticias y compartiéndolas por correo electrónico; (2) los políticamente alineados tanto hacia la izquierda como hacia la derecha son significativamente más proclives a compartir y comentar noticias en las redes sociales; y (3) las personas con alto interés en las noticias duras tienen significativamente más tendencia a comentar las noticias tanto en portales de noticias como en redes sociales.

Muchos académicos y profesionales creen que la cantidad de clics expresa de forma transparente el interés de los usuarios y la audiencia. Al analizar los datos de esta fuente, concluyen que los periodistas prefieren publicar información de relevancia sociopolítica y que el público busca, sobre todo, el entretenimiento (Boczkowski et al., 2011; Dick, 2011; Domingo, 2008; Lee et al., 2014; MacGregor, 2007; Robinson, 2011; Cantante, 2014; Thorson, 2008).

Sin embargo, como hemos señalado, otros autores han recurrido a diferentes indicadores para medir el interés de la audiencia, en lugar de solo tener en cuenta el número de clics. Dicen que "los clics solo cuentan una parte de la historia" (Kormelink y Costera Meijer, 2015, p. 14). Por ejemplo, los titulares pueden decir muy poco a los usuarios interesados en unos temas determinados, por lo que estos usuarios hacen clic en la noticia, mientras que otras veces las cabeceras les brindan suficiente información y no necesitan visitarlas.

Un ejemplo de los inicios referido a la naturaleza de los comentarios en los medios de comunicación online y su conexión con temas sociopolíticos (noticias duras) fue el sitio web BBC's Election 97, que mostró a los expertos políticos que la audiencia estaba muy interesada en los asuntos electorales (Ruggiero, 2000, p. 22).

Paradójicamente, las noticias que reciben más comentarios en los medios online no coinciden con las más visitadas, por lo que los temas sensacionales y los elementos que despiertan curiosidad obtienen muchos más clics, pero, por otro lado, los temas políticos y sociales reciben muchos más comentarios (Tenenboim y Cohen, 2015). En este contexto, los investigadores infieren que la decisión de escribir una historia y, por lo tanto, compartir ideas en un foro público, indica una preocupación especial por las noticias, por lo que los comentarios pueden entenderse como la expresión del interés por los asuntos públicos (Ksiazek, 2018).

Varias investigaciones han analizado los factores para explicar la cantidad de comentarios. El número de usuarios que realizan aportaciones en un artículo de noticias puede ser indicativo de la importancia, el interés o el efecto de dicho artículo (Tsagkias et al., 2009, p. 1765). Las informaciones negativas reciben muchos más comentarios que las positivas, y a los lectores no les gusta comentar las noticias insólitas, lo que puede deberse a que creen que la mayoría de dichas informaciones alteran los hechos (Liu et al., 2015, p. 774). Esto refuerza la idea de que los comentarios están especialmente relacionados con las noticias consideradas más relevantes.

En un estudio cualitativo, MacGregor (2007, p. 1097) entrevistó a periodistas de medios online y descubrió que los editores controlan los datos de la audiencia para volver a evaluar sus prioridades editoriales. Según este estudio, en respuesta a una historia con más clics, algunos periodistas ampliarían la cobertura, proporcionarían análisis adicionales o publicarían más información del mismo tipo.

En la era de Internet, los periodistas pueden saber muy rápidamente cómo está actuando la audiencia en relación a cuántos están leyendo una historia, qué historias prefieren o qué comentan $(\mathrm{Vu}, 2014$, p. 1094). La tecnología hace posible que las organizaciones de noticias registren el consumo de las mismas mediante el seguimiento de los clics y cuánto tiempo pasan los usuarios leyendo o viendo. 
Una conclusión generalizada es que la etiqueta de "noticias más vistas" incita a publicar un número mayor de temas relacionados con tales artículos, aunque los profesionales pueden sentir que basar su trabajo en este tipo de datos no está de acuerdo con sus demandas profesionales (Welbers et al., 2015).

\section{Metodología}

Este estudio acerca de las visitas y los comentarios y la relación de ambos elementos con las noticias duras y blandas en la red social de noticias Menéame se lleva a cabo con el objetivo de responder a estas dos preguntas principales:

Cuestión 1. ¿Qué tipo de noticias visitan más los usuarios, las duras o las blandas?

Cuestión 2. ¿Qué tipo de noticias comentan más los usuarios, las duras o las blandas?

Para responder a ambas preguntas, se han establecido dos hipótesis estrechamente relacionadas con las ideas desarrolladas en el marco teórico presentado anteriormente:

H1 Los usuarios prefieren noticias suaves para hacer clic.

H2. Los usuarios prefieren las noticias duras para comentarlas.

Aunque la red social Menéame emprendió su marcha en diciembre de 2005, el contador de clics comenzó a funcionar el 15 de septiembre de 2010 y, en consecuencia, este análisis incluye los siguientes cinco años hasta finales de 2015.

El universo de estudio consta de 51.520 noticias que aparecieron en la red durante ese período. Estas informaciones estaban recogidas en 2.576 portadas, cada una de las cuales contenía 20 noticias. Debido a esta peculiaridad, se ha utilizado el muestreo probabilístico por conglomerados para seleccionar las unidades: se eligieron al azar 186 portadas, que suman un total de 3.720 unidades de noticias. Esto significa que la muestra funciona con un nivel de confianza del $99 \%$ y un margen de error del $2 \%$.

Los datos de las dos variables fundamentales para el estudio se recogieron utilizando la información disponible en el propio sitio del agregador: a) la cantidad de comentarios realizados en cada noticia $\mathrm{y}, \mathrm{b})$ la cantidad de clics.

La tercera variable relevante, el tipo de noticia, obligó al equipo a llevar a cabo una laboriosa tarea para decidir cómo clasificar las informaciones. La propia red social requiere que el usuario que edita una noticia incluya la misma en una de las cuatro categorías de las que dispone, actualidad, ocio, cultura o tecnología, pero esta clasificación no era adecuada para el estudio. Por un lado, cada una de ellas acoge tanto noticias duras como blandas en diversos grados, mientras que distinguir entre ambas resultaba esencial en esta investigación. Por otro lado, los propios usuarios eligen la categoría para sus noticias, por lo que no se puede garantizar la fiabilidad de su codificación.

Teniendo en cuenta la importancia de clasificar adecuadamente las noticias duras y blandas, el equipo trabajó para establecer un conjunto convincente de categorías temáticas, elaborando una lista final de 14. Nueve de ellas tienen un amplio uso en las divisiones de temas usados en la publicación de medios de comunicación: Política, Economía, Justicia, Salud, Educación, Sociedad, Cultura y espectáculos, Ciencia y tecnología, y Deportes. Se seleccionaron cuatro más debido a su importancia 
en el contexto español de esos años (Corrupción, Inmigración, LGTBI y Violencia y discriminación contra la mujer). Todos ellos se completaron con la categoría "Otros". El índice Kappa dio una puntuación de 0,78 , por lo que el nivel de acuerdo entre los codificadores puede ser considerado como excelente, siguiendo la propuesta de Fleiss et al. (1981, p. 604).

A continuación, estas 14 categorías se redistribuyeron dependiendo de si eran noticias duras, blandas o generales, siguiendo la metodología propuesta por Lehman-Wilzig y Seletzky (2010), quienes argumentan la necesidad de crear un tercer tipo intermedio porque el reservado para las blandas ha incluido, hasta la fecha, bloques temáticos muy heterogéneos. La distribución final fue la siguiente: a) Noticias duras: Política, Economía, Justicia, Educación, Corrupción, LGBT, Inmigración, Violencia y discriminación contra la mujer; b) Noticias blandas: Sociedad, Cultura y espectáculos, Deportes, Otros; c) Noticias generales: Ciencia y tecnología, Salud.

\section{Resultados}

\subsection{Qué tipo de noticias visitan más los usuarios}

La red social Menéame muestra el número de clics que recibe cada noticia, lo que sirve para establecer la cantidad de visitas que ha tenido esa pieza informativa. Los clics son un indicador de las preferencias de la mayoría de los usuarios como consumidores, en oposición al número de comentarios. Estos últimos, en cambio, reflejan el grado de interés de los miembros más activos por los problemas sociopolíticos.

Tabla 1. Noticias por tema y tipo ordenadas en base a la media de visitas.

\begin{tabular}{|l|r|r|r|l|}
\hline Categoría temática & \multicolumn{1}{|c|}{$\begin{array}{c}\text { Número de } \\
\text { noticias }\end{array}$} & \multicolumn{1}{c|}{ Clics } & Media & Tipo \\
\hline Otros & 13 & 136.716 & $10.516,6$ & Blanda \\
\hline Sociedad & 750 & 7.478 .077 & $9.970,8$ & Blanda \\
\hline Cultura y espectáculos & 258 & 2.316 .598 & $8.979,1$ & Blanda \\
\hline Ciencia y tecnología & 451 & 3.449 .751 & $7.649,1$ & General \\
\hline Educación & 73 & 547.177 & $7.495,6$ & Dura \\
\hline Deportes & 60 & 405.917 & $6.765,3$ & Blanda \\
\hline Violencia y discriminación contra la mujer & 47 & 305.021 & $6.489,8$ & Dura \\
\hline Economía & 510 & 3.206 .690 & $6.287,6$ & Dura \\
\hline LGTB & 24 & 146.642 & $6.110,1$ & Dura \\
\hline Salud & 88 & 527.316 & $5.992,2$ & General \\
\hline Inmigración & 18 & 105.867 & $5.881,5$ & Dura \\
\hline Política & 1.062 & 5.955 .405 & $5.607,7$ & Dura \\
\hline Corrupción & 270 & 1.192 .421 & $4.416,4$ & Dura \\
\hline Justicia & 96 & 299.471 & $3.119,5$ & Dura \\
\hline Total & 3.720 & 26.073 .069 & $7.008,9$ & \\
\hline
\end{tabular}

Fuente: elaboración propia.

Tres categorías temáticas, clasificadas como noticias blandas, reciben un número mayor de visitas. La categoría "Otros", de carácter irrelevante y compuesta principalmente por historias humorísticas o 
de contenido excéntrico, se sitúa en el primer puesto, con un promedio de 10.516 visitas. A continuación, le siguen dos categorías: "Sociedad" y "Cultura y espectáculos". Por el contrario, la mayoría de las categorías correspondientes a noticias duras recibe menos visitas: "Justicia", ubicada al final de la lista, es la categoría que obtiene el promedio más bajo, con casi 7.400 clics menos que "Otros". Las categorías incluidas en las noticias generales, "Ciencia y Tecnología" y "Salud" se encuentran en la mitad de la lista.

Tabla 2. Noticias duras, blandas y generales y media de clics.

\begin{tabular}{|l|r|r|r|}
\hline & Número de noticias & \multicolumn{1}{c|}{ Clics } & \multicolumn{1}{c|}{ Media } \\
\hline Noticias duras & 2.100 & $11.758,694$ & $5.599,4$ \\
\hline Noticias blandas & 1.081 & $10.337,308$ & $9.562,7$ \\
\hline Noticias generales & 539 & $3.977,067$ & $7.378,6$ \\
\hline Total & 3.720 & $26.073,069$ & $7.008,9$ \\
\hline
\end{tabular}

Fuente: elaboración propia.

El resumen en la tabla 2 muestra que las noticias blandas reciben en promedio casi 4.000 clics más que las noticias duras $(9.562,7$ y $5.599,4$ clics, respectivamente).

\subsection{Qué clase de noticias comentan más los usuarios}

Seis categorías temáticas incluidas en el tipo de noticias duras obtienen el mayor número de comentarios. Son Violencia y discriminación contra la mujer, LGTB, "Educación", "Política", "Economía" e "Inmigración".

Tabla 3. Noticias por categoría temática y tipo, ordenadas en base a la media de comentarios.

\begin{tabular}{|l|r|r|r|l|}
\hline \multicolumn{1}{|c|}{ Categoría } & \multicolumn{1}{c|}{ Casos } & \multicolumn{1}{c|}{ Comentarios } & \multicolumn{1}{c|}{ Media } & \multicolumn{1}{c|}{ Tipo } \\
\hline Violencia y discriminación contra la mujer & 47 & 4.562 & 97,1 & Dura \\
\hline LGTB & 24 & 2.270 & 94,6 & Dura \\
\hline Educación & 73 & 6.884 & 94,3 & Dura \\
\hline Política & 1.062 & 94.508 & 89 & Dura \\
\hline Economía & 510 & 41.338 & 81,1 & Dura \\
\hline Inmigración & 18 & 1.373 & 76,3 & Dura \\
\hline Deportes & 60 & 4.290 & 71,5 & Blanda \\
\hline Justicia & 96 & 6.842 & 71,3 & Dura \\
\hline Sociedad & 750 & 52.339 & 69,8 & Blanda \\
\hline Salud & 88 & 6.122 & 69,6 & General \\
\hline Cultura y espectáculos & 258 & 17.795 & 69 & Blanda \\
\hline Corrupción & 270 & 17.193 & 63,7 & Dura \\
\hline Ciencia y tecnología & 451 & 24.561 & 54,5 & General \\
\hline Otros & 13 & 670 & 51,5 & Blanda \\
\hline Total & 3.720 & 280.747 & 75,5 & \\
\hline
\end{tabular}

Fuente: elaboración propia. 
En el otro extremo, la categoría de blanda, "Otros", que recibió la mayor cantidad de visitas, obtiene el menor número de comentarios en promedio $(51,5)$ mientras que "Política", incluida en la categoría de noticias duras, que había sido la tercera con menos clics es, al contrario, la cuarta con más comentarios.

Tabla 4. Media de comentarios por tipo de noticia.

\begin{tabular}{|l|r|r|r|}
\hline \multicolumn{1}{|c|}{ Tipo } & \multicolumn{1}{c|}{ Cantidad } & Comentarios & Media \\
\hline Noticias duras & 2.100 & 174.970 & 83,3 \\
\hline Noticias blandas & 1.081 & 75.094 & 69,5 \\
\hline Noticias generales & 539 & 30.683 & 56,9 \\
\hline Total & 3.720 & 280.747 & 75,5 \\
\hline
\end{tabular}

Fuente: elaboración propia.

En la tabla 4 se puede apreciar una clara diferencia entre los dos tipos de noticias. En promedio, las noticias duras obtienen más comentarios que las noticias blandas ( 83.3 y 69.5 respectivamente) y las noticias generales son las que reciben menos comentarios (75.5).

\subsection{Relación entre clics y comentarios}

Se puede afirmar que los clics y los comentarios funcionan de manera opuesta. Como se ve en el gráfico 1, si correlacionamos el promedio de comentarios de las categorías temáticas con su promedio de clics, el resultado es $\mathrm{R}=-0.31$. Esto significa que hay una correlación negativa, con una fuerza moderada, que muestra esta tendencia: un aumento en los clics significa una disminución en los comentarios, y viceversa.

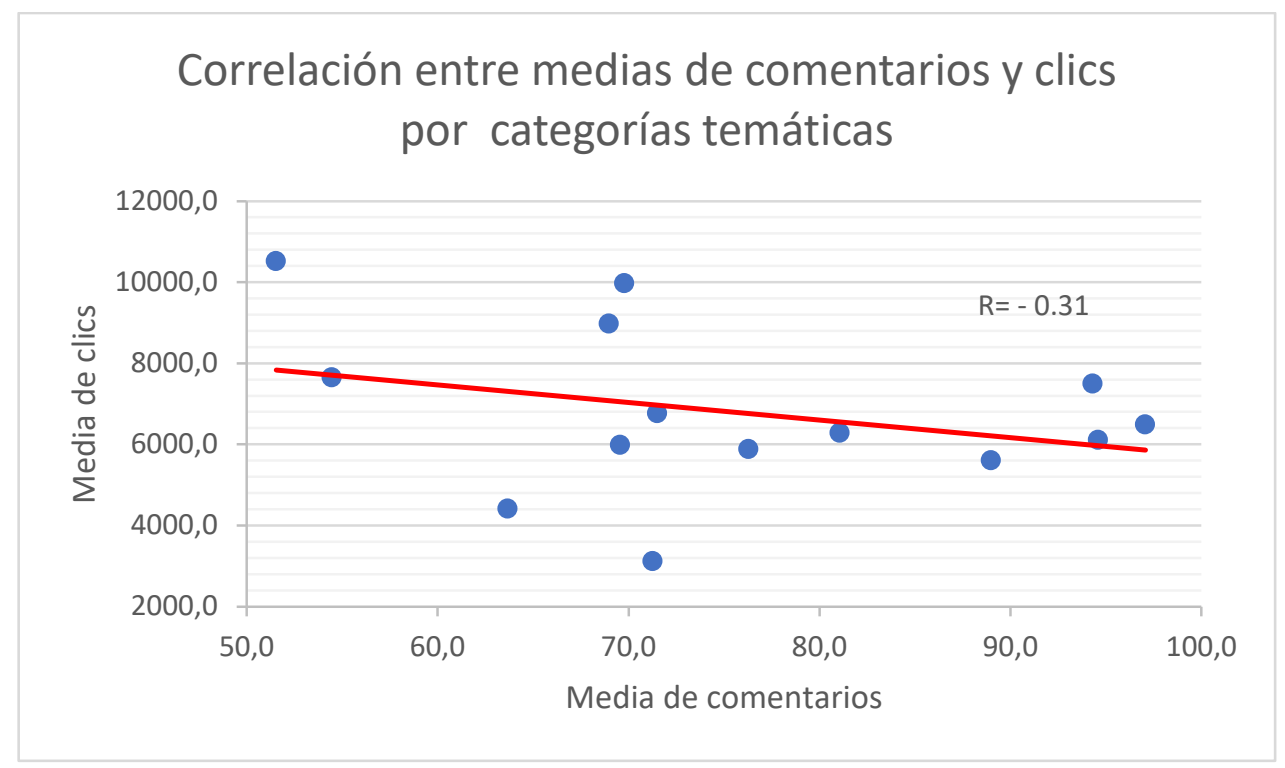

Gráfico 1: correlación entre medias de comentarios y clics de las categorías temáticas en las noticias de portada de Menéame.

Fuente: elaboración propia.

Esta correlación negativa nos lleva a la conclusión de que los comentarios y los clics son indicadores de la existencia de intereses muy diferentes entre los usuarios de la red. El interés por los asuntos públicos, expresado por los comentarios, tiende a funcionar sin tener en cuenta el interés por el entretenimiento, manifestado por los clics. 


\section{Discusión y conclusiones}

Los resultados nos muestran que los clics y los comentarios son indicadores de dos tipos de intereses por parte de los lectores. Si examinamos estas motivaciones basándonos en la teoría de usos y gratificaciones, la conclusión es que los clics están más conectados con el entretenimiento, mientras que los comentarios dejan entrever un mayor interés en la participación en asuntos públicos.

Los resultados señalan que ambos elementos funcionan con una lógica opuesta en la red social Menéame. La tendencia es clara: a medida que aumentan los comentarios, los clics disminuyen, y viceversa. Mediante la proyección de este resultado al campo de los medios de comunicación se deduce que no es conveniente que los periodistas confíen solo en uno de esos elementos -sobre todo en los clics- para tomar decisiones sobre el control y la edición. Los usuarios pueden visitar masivamente varios tipos de noticias, pero, en realidad, la acción de comentar indica una mayor preocupación e interés por los asuntos públicos. El hecho de que las noticias duras reciban más comentarios nos lleva a pensar que los criterios utilizados por los usuarios para valorar las noticias relevantes están más cerca de los que tradicionalmente se atribuyen a los periodistas.

Contrariamente a esto, los clics manifiestan una tendencia al entretenimiento y al escapismo. Los usuarios disfrutan de la sensación de ocio otorgada, especialmente, por las noticias blandas, sabiendo que han sido promovidas con los votos de otros usuarios, por lo que, presumiblemente, incluso estas noticias pueden llegar a ofrecer alguna garantía de que respetarán varios requisitos básicos.

La comunidad de usuarios de la red social de noticias Menéame se mueve en un dilema permanente: elegir entre el compromiso con las noticias más relevantes desde el punto de vista sociopolítico y la incursión en las noticias que fomentan el escapismo. Las primeras valen para comprometerse en una mayor participación, mientras que las últimas solo merecen un clic. En nuestra opinión, se necesitan más investigaciones para probar si estas características asociadas a esta comunidad son comunes en otras redes sociales.

\section{Referencias}

Boczkowski, P. J., Mitchelstein, E. \& Walter, M. (2011). Convergence Across Divergence: Understanding the Gap in the Online News Choices of Journalists and Consumers in Western Europe and Latin America. Communication Research, 38(3), 376-396.

Carazo, C. (2018). Periodismo y agenda setting: una discusión sobre el interés por asuntos públicos de la ciudadanía. Revista de Ciencias Sociales, 160, 15-35.

Diakopoulos, N. \& Naaman, M. (2011). Towards Quality Discourse in Online News Comments. Proceedings of the ACM 2011 Conference on Computer supported cooperative work, 133-142.

Dick, M. (2011). Search engine optimisation in UK news production. Journalism Practice, 5(4), 462-477.

Domingo, D. (2008). Interactivity in the daily routines of online newsrooms: dealing with an uncomfortable myth. Journal of Computer-Mediated Communication, 13(3), 680-704.

Fleiss, J. L., Levin, B. \& Cho Paik, M. (2003). Statistical Methods for Rates and Proportions. Wiley \& Sons. 
Kalogeropoulos, A., Negredo, S., Picone, I. \& Nielsen, RK. (2017). Who Shares and Comments on News?: A Cross-National Comparative Analysis of Online and Social Media Participation. Social Media + Society, October-December, 1-12.

Katz, E., Blumler, J. \& Gurevitch, M. (1974). Utilization of mass communication by the individual. In J. Blumler \& E. Katz (Eds.), The uses of mass communication: Current perspectives on gratifications research (pp. 19-34). Sage.

Katz, E. \& Foulkes, D. (1962). On the use of mass media as escape: Clarification of a concept. Public Opinion Quarterly, 26, 377-388.

Katz, E., Gurevitch, M. \& Haas, H. (1973). On the use of the mass media for important things. American Sociological Review, 38, 164-181.

Kilgo, Harlow, García-Perdomo, Salaverría (2018). A new sensation? An international exploration of sensationalism and social media recommendations in online news publications. Journalism, 19(11), 1497-1516.

Lee, A. M., Lewis, S. C. \& Powers, M. (2014). Audience Clicks and News Placement: A Study of Time-Lagged Influence in Online Journalism. Communication Research, 41(4), 505-530.

MacGregor, P. (2007). Tracking the online audience: Metric data start a subtle revolution. Journalism Studies, 8(2), 280-298.

McCombs, M. (2005). A Look at Agenda-setting: past, present and future. Journalism Studies, 6(4), 543-557. https://doi.org/10.1080/14616700500250438

Liu, Q., Zhou, M. \& Zhao, X. (2015). Understanding News 2.0: A framework for explaining the number of comments from readers on online news. Information \& Management, 52, 764-776.

Robinson, S. (2011). Journalism as process: The organizational implications of participatory online news. Journalism \& Communication Monographs, 13(3), 137-210.

Ruggiero, T. E. (2000). Uses and Gratifications Theory in the 21 st Century. Mass communication \& Society, 3(1), 3-37.

Singer, J. B. (2014). User-generated visibility: Secondary gatekeeping in a shared media space. New media \& Society, 16(1), 55-73.

Thorson, E. (2008). Changing patterns of news consumption and participation: News recommendation engines. Information, Communication \& Society, 11(4), 473-489.

Tsagkias, M., Weerkamp, W. \& de Rijke, M. (2009). Predicting the Volume of Comments on Online News Stories. Proceedings of the 18th ACM conference on Information and knowledge management, 1765-1768.

$\mathrm{Vu}, \mathrm{H}$. T. (2014). The online audience as gatekeeper: The influence of reader metrics on news editorial selection. Journalism, 15(8), 1094-1110. 


\section{AUTORES:}

\section{Begoña Zalbidea Bengoa}

Departamento de periodismo. Universidad del País Vasco/Euskal Herriko Unibertsitatea. España.

Doctora en Periodismo y Titular de Universidad, posee las licenciaturas en Ciencias de la Información y en Derecho. Premio Extraordinario de Doctorado, antes de dedicarse a la docencia ejerció el periodismo en distintos medios de comunicación. Entre 2007 y 2011 dirigió Zer. Revista Científica de Comunicación Social. En la actualidad es miembro de su Consejo Editorial, así como de los comités científicos de Comunicació. Revista de recerca i d'anàlisi y Fonseca. Su actividad docente e investigadora se ha centrado, fundamentalmente, en la ética y deontología de los profesionales y de los medios, en el análisis de contenido, en la especialización periodística y en la historia del periodismo. Ha estado como profesora visitante en distintas universidades latinoamericanas, donde ha impartido clases en másteres y en cursos de formación dirigidos a docentes y profesionales de la comunicación. Es Directora del Departamento de Periodismo, miembro de la Comisión de Postgrado y de la Junta de Campus de Bizkaia de la UPV/EHU, así como de la Junta y Comisión Permanente de la Facultad de Ciencias Sociales y de la Comunicación. Dirige el grupo de investigación Ética e Información. Además de participar en otros proyectos, figura como investigadora principal en tres subvencionados por el Ministerio de Ciencia e Innovación, y en un cuarto de la UPV/EHU. Su labor divulgativa se recoge en más de 40 títulos y en decenas de intervenciones en congresos nacionales e internacionales.

bego.zalbidea@ehu.eus

\section{Índice H: 7}

Orcid ID: https://orcid.org/0000-0001-9518-5180

Google Scholar: $\underline{\text { https://scholar.google.es/citations?hl=es\&user=5uyNX7MAAAAJ }}$

\section{Santiago Urrutia}

Departamento de Sociología y Trabajo Social. Universidad del País Vasco/Euskal Herriko Unibertsitatea. España.

Licenciado en CC. PP. y Sociología por la Universidad de Deusto (1983) y doctor por la UPV/EHU (1999). Ha sido responsable del Máster de Comunicación Multimedia UPV/EHU-EITB (2014-2017) y en la actualidad forma parte de su comisión académica. Su labor docente se centra, fundamentalmente, en métodos de investigación aplicados a la comunicación. Ha realizado estancias como investigador visitante en las Universidades de Reno y en la Universidad de Glasgow. Sus principales líneas de investigación son: 1) Medios de comunicación y periodismo en lenguas minoritarias europeas; 2) Ética de los medios, y en especial el análisis de las representaciones visuales de sexo y violencia. En lo que respecta a la primera línea de investigación ha formado parte del proyecto y grupo de investigación 'European Minority Language Media and Journalism Research Group', dirigido por el Prof. Iñaki Zabaleta. Con respecto a la segunda, está integrado en el grupo de investigación Ética y Excelencia Informativa, dirigido por la Prof. Begoña Zalbidea. Ha participado en alrededor de 50 comunicaciones presentadas a congresos nacionales e internacionales y 25 artículos y capítulos de libros publicados en revistas y publicaciones. Cuenta con un sexenio de investigación reconocido por la CNEAI.

santi.urrutia@ehu.eus

Índice $\mathrm{H}: 6$

Orcid ID: https://orcid.org/0000-0002-0571-3363

Google Scholar:

https://scholar.google.es/citations?+user=ShqcOJcAAAAJ\&hl=es\&pli=1\&user=2 IgYxsAAAAJ 


\section{Idoia Camacho-Markina}

Departamento de Periodismo. Universidad del País Vasco/Euskal Herriko Unibertsitatea. España.

En la actualidad es Vicedecana de Comunicación y Relaciones Externas de la Facultad de Ciencias Sociales y de la Comunicación de la Universidad de del País Vasco/Euskal Herriko Unibertsitatea. Es Licenciada (1996) y Doctora (2002) en Ciencias de la Información por la UPV/EHU y Profesora Agregada en la Facultad de Ciencias Sociales y de la Comunicación de esta universidad. Ha sido Responsable del Programa de Doctorado en Comunicación Social (2009-2017) y ha dirigido la revista científica ZER. Revista de Estudios de Comunicación (2014-2018). Cuenta con 2 sexenios reconocidos por la CNEAI. Forma parte del grupo de investigación Ética, Información y Ciudadanía (GEIC), que está llevando adelante el Proyecto de Investigación "Reacciones desde la ética ciudadana en la red social 'Menéame' ante los contenidos de los medios de comunicación convencionales y sociales en España", financiado por el Ministerio de Economía y Competitividad del Gobierno de España. Ha participado en proyectos de investigación financiados de forma ininterrumpida desde diciembre de 2006, en uno de los cuales ha sido la investigadora principal: "La información de salud en la prensa diaria vasca". Dirige 3 tesis doctorales y es autora del libro Formación de portavoces: Cómo conseguir una comunicación efectiva ante los medios y otros públicos y de publicaciones sobre el tratamiento de diversos temas sanitarios en la prensa, la función educativa de los medios de comunicación y los gabinetes de comunicación en la administración local, entre otras.

idoia.camacho@ehu.eus

Índice H: 10

Orcid ID: https://orcid.org/0000-0003-2296-9472

Google Scholar: https://scholar.google.es/citations?user=ShqcOJcAAAAJ\&hl=en

\section{José María Pastor González}

Departamento de Periodismo. Universidad del País Vasco/Euskal Herriko Unibertsitatea. España. Jose Mari Pastor González es profesor del Departamento de Periodismo de la Facultad de Ciencias Sociales y de la Comunicación de la Universidad del País Vasco (UPV/EHU). Licenciado en Periodismo en esta misma facultad, se doctoró en la UPV con la tesis La Información política vasca en el informativo Gaur Egun de ETB (Televisión Vasca). Trabaja en la Universidad del País Vasco desde 2007, donde imparte docencia de Redacción Informativa en Prensa y de Géneros Interpretativos. En los últimos cinco años ha formado parte de cuatro proyectos de investigación y ha publicado artículos en diversas revistas de estudios sociales y de comunicación. En la actualidad es miembro del Grupo Consolidado Ética, Información y Ciudadanía (GEIC). Pastor comenzó su andadura en los medios de comunicación en 1981, en la revista Anaitasuna y posteriormente en el suplemento Egunon del diario Egin. Trabajó en Euskal Telebista, (1985-1986 y 1988-1991)) y en Euskaldunon Egunkaria y Berria, únicos diarios editados en lengua vasca, hasta el año 2007. Siendo redactor jefe de Internacional en este último periódico viajó a Irlanda del Norte, Argelia, Sáhara Occidental, Irak, Serbia, Montenegro, Israel y Palestina, entre otros. Ha sido galardonado con el premio de periodismo Rikardo Arregi (2004), el de Prensa de los premios Argia del mismo año como reconocimiento al trabajo de la sección de Internacional del diario Berria y, por último, con el Joan Cendrós (2006), por la cobertura informativa de las elecciones catalanas del mismo año. josemari.pastor@ehu.eus

\section{Índice $\mathrm{H}: 1$}

Orcid ID: https://orcid.org/0000-0003-4687-7935

Google Scholar: https://scholar.google.com/citations?user=m8WhAQ0AAAAJ\&hl=es 